\title{
Multi-objective optimization of the UAV wing by means of evolutionary computations
}

\author{
A. Długosz*, W. Klimek** \\ *Institute of Computational Mechanics and Engineering, Faculty of Mechanical Engineering Silesian University of \\ Technology, Gliwice, Poland, E-mail: adam.dlugosz@polsl.pl \\ **Flytronic Sp.zo.o. Gliwice, Poland,E-mail: wiktorklimek@gmail.com \\ cross ${ }^{\text {ref }}$ http://dx.doi.org/10.5755/j01.mech.22.6.14419
}

\section{Introduction}

During the design process of the aircraft wing, several goals and restrictions have to be considered. The wings/aerofoils should be characterized by: high lifting force, low aerodynamics drag, low mass, high endurance, etc. Such criteria are usually contradictory. In order to take into consideration more than one criterion, efficient multiobjective optimization method have to be applied [1]. Moreover for such problems criteria calculated on the basis of results derived from numerical simulations may have many local minima. It complicates greatly solving optimization problems. In a classic approach deterministic algorithms are used. There are mainly two groups: non-gradient methods (e.g. Powell, Nelder-Mead algorithms) and gradient methods (e.g. steepest descent, conjugate gradient, Quasi-Newton methods). The second group is more effective numerically, but requires knowledge of the gradient of the objective function. For many practical problems calculation of the gradient is very difficult. Additionally, the disadvantage of any deterministic methods is their tendency to locate local extremes. In the case of solving optimization for multiple criteria, achieving a solution by these methods becomes even more difficult. Optimization task with multiple criteria in the classical sense most often rely on their transition to the single objective problem (weighting sum method, $\varepsilon$-constrained method, etc.). These methods are easy to use, but have several drawbacks and limitations. One is the need for additional information before optimization (which can be difficult without prior knowledge of the problem) and the ability of achieving only one solution as a result of a single optimization procedure. The population bio-inspired algorithms, like evolutionary algorithms [2], do not have above drawbacks. They allow most of all for: the possibility of achieving not one but a set of Pareto-optimal solutions in the single optimization task, the possibility of use for multimodal and discontinuous functions, including constraints of any kind and no need for calculating the gradients of the objective function.

The problem of optimal design of the wing as well for passenger or military aircraft, as for Unmanned Aerial Vehicle (UAV), has been considered by many researchers [3-5]. Different methods (bio-inspired techniques or gradient based algorithms) have been used for single and multiobjective optimization, only for properties of the composite materials [6-10], as well as for properties of entire components $[11,12]$. The paper presents an application of the inhouse implementation of the evolutionary multi-objective algorithm in optimization of the UAV wing part. Proposed algorithm have been tested on several mathematical benchmark problems, showing its superiority on NSGAII (one of the most popular multi-objective evolutionary algorithm). Moreover, proposed algorithm has been successfully applied in the optimization of different mechanical structures [13]. Functionals, which represent each criteria, are calculated by means of Finite Element Method (FEM) [24]. The wing used for UAVs are modelled, tested and optimized. The wing used for testing was manufactured especially for this purpose and based on typical UAV wing geometry Fig. 1, a presents part of the wing, cut from the real structure. Numerical model of the wing composed of different composite materials is prepared $[15,16]$. Experiment of the three-point bending test on the part of the wing has been performed by means of universal testing machine. The results of the numerical simulation are compared with the results from the experiment. In the optimization task, the invariable aerodynamic properties of the airfoil are assumed (the external shape of the wing is given and it does not change during optimization). The aim of the optimization is to improve strength and stiffness together with reduction of the weight of the structure. Three different types of functionals, which depend on stress, stiffness and the total mass are defined. Values of the functionals are calculated on the basis of results, obtained from FEM analyses. FEM software MSC Patran and Nastran are used. In order to build separate boundary-value problems automatically, additional ad-hoc software and proper scripts are prepared. Examples of multiobjective optimization by means of Pareto-optimal set of solutions are presented.

\section{Numerical model and the experiment}

The UAV wing, based on typical geometry has been taken for testing. Fig. 1, a presents the part, which has been cut from the wing.
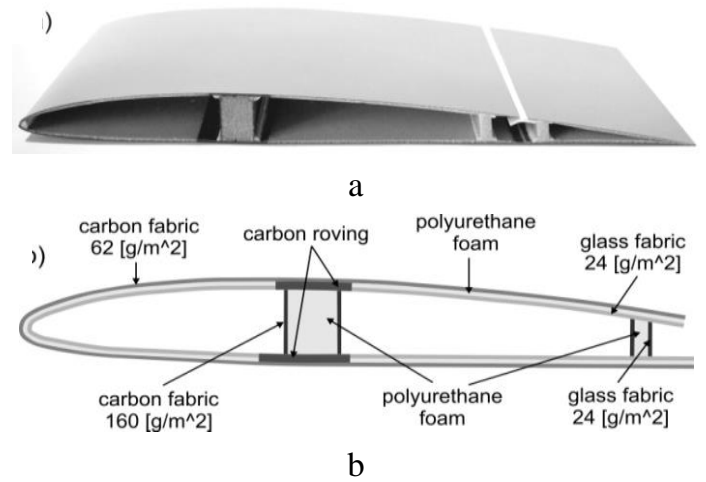

Fig. 1 a - Part of the wing; $b$ - distribution of the materials 
The structure consists of: polyurethane foam, carbon and glass fabric. Connection between girder and the outer panel are made by using additional woven roving (Fig. 1, b). The aileron is not considered in the numerical model and in the experiment, because its effect on the load transfer can be negligible.

The three-point bending test on the structure is performed by means of universal testing machine (MTS Insight System). The maximum force $F_{\max }=989 \mathrm{~N}$ has been measured for the deflection equal to $u\left(F_{\max }\right)=0.734 \mathrm{~mm}$. Therefore, stiffness of the structure $(K=1347 \mathrm{~N} / \mathrm{mm})$ is calculated from the following formula:

$$
K=\frac{F_{\max }}{u\left(F_{\max }\right)}
$$

Fig. 2 presents installation of the wing in the machine and the appearance of the damaged item after the experiment.
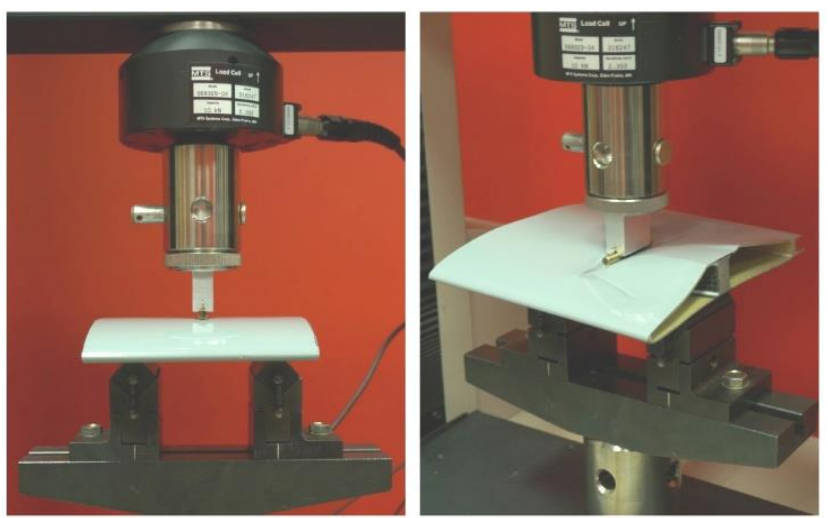

Fig. 2 Part of the wing installed in the MTS Insight machine and the damaged item after the experiment

Numerical model of the considered part of the wing is prepared on the basis of the real structure. Linearelastic constitutive model of structure consist of composite materials is considered [17-20]. Four materials with the orthotropic properties (carbon fabric $62 \mathrm{~g} / \mathrm{m}^{2}$, carbon fabric $160 \mathrm{~g} / \mathrm{m}^{2}$, glass fabric $24 \mathrm{~g} / \mathrm{m}^{2}$ and roving) and one isotropic (polyurethane foam PUR) are defined. Such materials are used to define four laminates which creates the model of the wing. The location of the particular laminates is presented in Fig. 3.

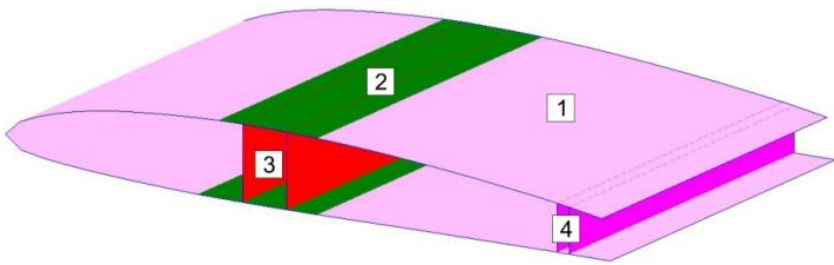

Fig. 3 Laminates used in the construction of the wing: 1 - carbon fabric $62 \mathrm{~g} / \mathrm{m}^{2}$, foam, glass fabric $24 \mathrm{~g} / \mathrm{m}^{2}$; 2 - carbon fabric $62 \mathrm{~g} / \mathrm{m}^{2}, \quad$ roving; 3 - carbon fabric $160 \mathrm{~g} / \mathrm{m}^{2}$, foam; 4 - glass fabric $24 \mathrm{~g} / \mathrm{m}^{2}$, foam

The numerical model of the wing is built in MSC Patran system. The model is discretized by means of Quad4 shell elements (total number of DOF is about 6500). In order to correctly represent load in the model, additionally multi- point constrained elements (MPC) is used (Fig. 4). Linear static analysis is performed by means of MSC Nastran system. Fig 5 presents the deformation map ( $\mathrm{Z}$ component of the displacement) for the model. The maximum value of the displacement is $0.882 \mathrm{~mm}$. Therefore, the stiffness of the structure calculated from the formula (1) is equal to $1124 \mathrm{~N} / \mathrm{mm}$. The difference between stiffness, calculated on the basis of experiment, and the stiffness calculated on the basis of results derived from numerical simulation is $17 \%$. Such discrepancy can be treated as satisfactory taking into account many simplifications in the numerical model.

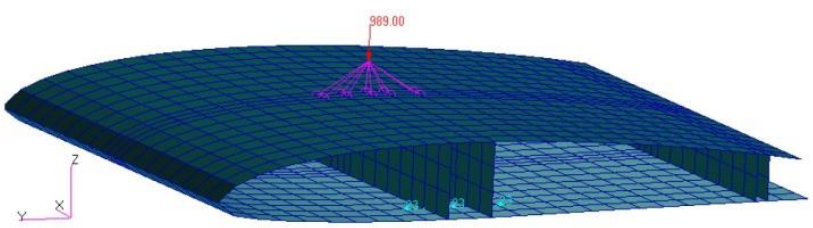

Fig. 4 Finite element mesh and boundary conditions for the model by means of MPC

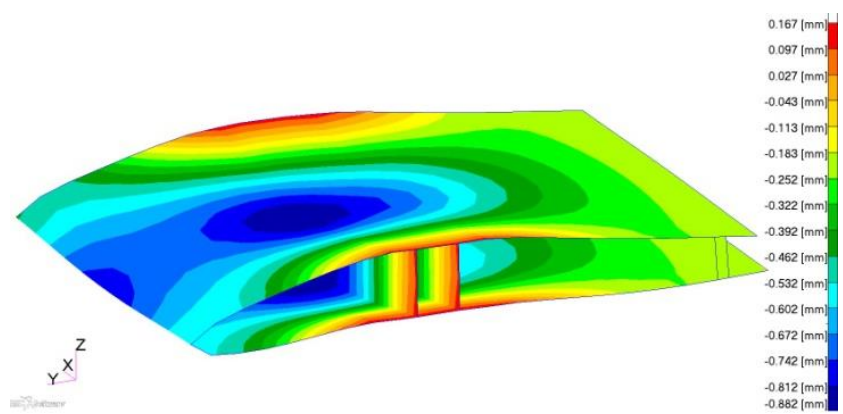

Fig. 5 The map of vertical displacements in the model

\section{Formulation of the multi-objective optimization problem}

As mentioned in chapter 1 optimal design of complex mechanical structures requires consideration of many criteria. In practice, this requires coupling of appropriate multi-criteria optimization methods and different numerical simulation techniques. Solution of the multi-criteria optimization problem is to find the vector of design variables, representing e.g. the geometry, material properties, boundary condition values, material costs, etc., for which defined objective functions achieve extremes. The optimization problem can be formulated as a minimization or maximization tasks. For the minimization case, the vector of design variables $\boldsymbol{x}=\left[x_{1}, x_{2}, \ldots, x_{n}\right]$, which minimize set of $k$ objective functions $f(\boldsymbol{x})=\left[f_{1}(\boldsymbol{x}), f_{2}(\boldsymbol{x}), \ldots, f_{n}(\boldsymbol{x})\right]$ is searched for. In general, the tasks included additional equality and inequality constraints, are imposed on the design variables. Thus for the multi-criteria optimization the decision space is $n$-dimensional, whereas objective space is $k$-dimensional. The concept of Pareto uses terms such as: dominated solutions, non-dominated solutions (Pareto-optimal). Taking into consideration two vectors $\boldsymbol{x}$ and $\boldsymbol{y}$ in the admissible solution space:

- strongly dominates $\boldsymbol{y}$, if:

$$
\forall i \in\{1,2, \ldots, k\}: f_{i}(\boldsymbol{x})<f_{i}(\boldsymbol{y}) \text {; }
$$


$\left.\begin{array}{l}\forall i \in\{1,2, \ldots, k\}: f_{i}(\boldsymbol{x}) \leq f_{i}(\boldsymbol{y}) \\ \wedge \quad \exists j \in\{1,2, \ldots, k\}: f_{j}(\boldsymbol{x})<f_{j}(\boldsymbol{y})\end{array}\right\} ;$

- $\boldsymbol{x}$ is neutral (incomparable) relative to $\boldsymbol{y}$, if:

$\exists i, j \in\{1,2, \ldots, k\}: f_{i}(\boldsymbol{x})<f_{i}(\boldsymbol{y}) \wedge f_{j}(\boldsymbol{y})>f_{j}(\boldsymbol{x})$.

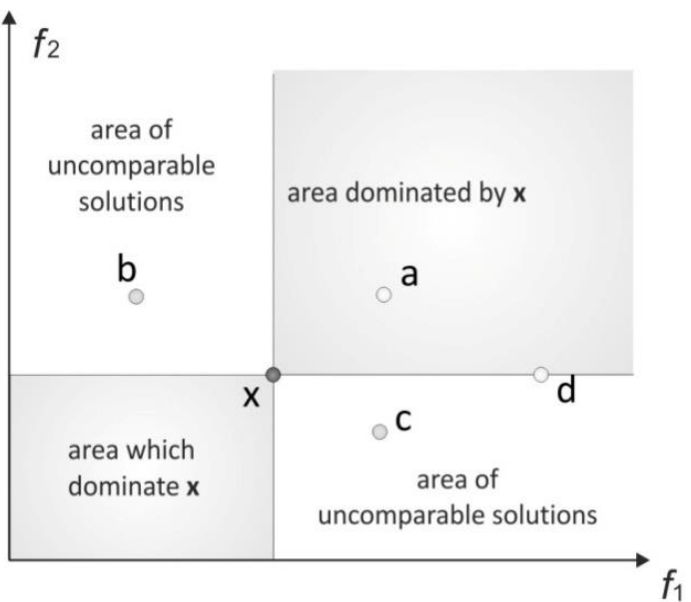

Fig. 6 Relation between solutions in the search space

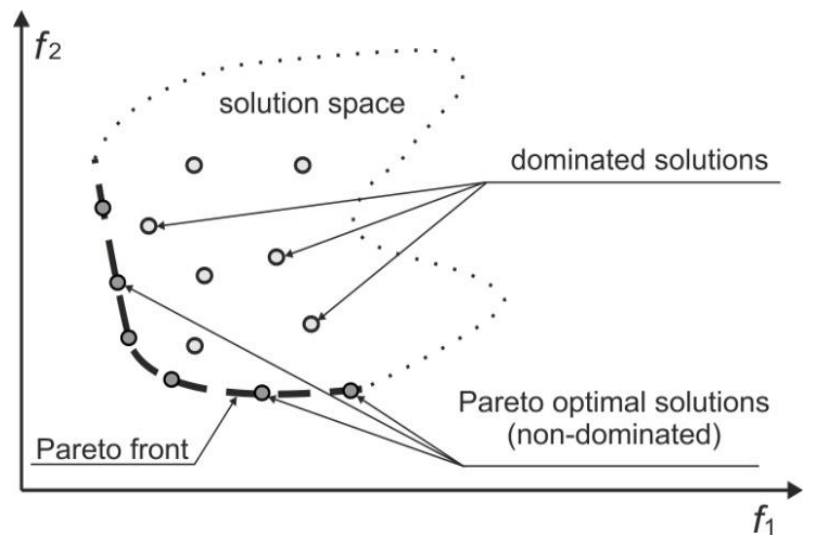

Fig. 7 Representation of the Pareto front for the bi-objective case

Fig. 6 shows the relationship between the vectors of solutions. Solution $\boldsymbol{a}$ is dominated by $\boldsymbol{x}$ (in other words solution $\boldsymbol{x}$ dominates $\boldsymbol{a}$, solution $\boldsymbol{x}$ weakly dominates solution $\boldsymbol{d}$, whereas solutions $\boldsymbol{b}$ and $\boldsymbol{c}$ are neutral (incomparable) relative to $\boldsymbol{x}$. The set of all Pareto-optimal solutions creates a so-called. Pareto front (Fig. 7). If the three criteria are considered, the solutions belonging to the Pareto produce surfaces. Optimization method based on Pareto approach belong to the group of a posteriori methods. On the basis of a set of solutions it is possible to select solution according to the established preferences. Optimization tasks with multiple criteria can be also solved, assuming before optimization certain assumptions concerning, e.g. preferences which relate to each criterion. (a priori methods). In most cases, these methods involve the transformation task with multiple criteria to the single-objective optimization problems. For this purpose, the most widely used approach is the weighted sum method. These methods are easy to use because they do not require modification of the optimization algorithm (the single-objective optimization algorithm can be used). However, they have significant limitations, which reduces the scope of their application.
It should be emphasized that the set of final solutions obtained by algorithms which utilizes Pareto approach, may include additional information about the nature of the conflict between the criteria (small change in the some areas of the front for one criterion causes a dramatic change on the other, there are areas of discontinuity on the front, etc.). Selection of the optimal solution apart from accepted user's preferences may also involve such information. Moreover, advantage of this approach is to obtain a set of Pareto-optimal solutions as a result of a run the single optimization task. In-house implementation of the Multi-Objective OPTIMization tool based on evolutionary algorithm (MOOPTIM) is used for optimization. The proposed algorithm is similar to NSGAII. It uses nondominated sorting procedure for classification of the individuals in population and a crowding coefficient to preserve diversity in the population $[21,22]$. The main difference between MOOPTIM and NSGA II is based on changes in selection mechanism and the application of different evolutionary operators. The proposed implementation has more evolutionary operators comparing to the NSGAII. Two types of mutation (uniform and Gaussian once) and two types of crossover operators (simple and arithmetical) are used. It should be emphasized that Gaussian mutation has significant influence on the effectives of searching by the algorithm. This operator requires an extra parameter (besides mutation probability) in the form of the mutation range (from 0 to 1 ). It was observed that higher values of the probability and range of this operator improved the convergence of the algorithm, especially for more difficult tasks. The other difference between these algorithms is related to the formation of offspring population. There is no binary tournament selection operator in MOOPTIM, but individuals are selected on the basis of nondomination level and crowding coefficient. Detailed description of the MOOPTIM algorithm can be found in [13]. MOOPTIM was tested on several benchmarks (SCH, ZDT1, ZDT2, ZDT3, ZDT4, ZDT6, CONSTR, SRN, TNK) and also on some engineering problems. The results obtained using MOOPTIM in most cases are better in comparison with the results obtained by means of NSGA-II.

\section{Multi-objective optimization of the model of the wing}

Chromosomes genes represent the vector of design variables $\boldsymbol{x}$ in optimization task. The box constraints are imposed on each gene. Eleven design variables are used for preparation of the geometry of the structure. They are responsible for: the position of the girders ( $V 1$ and $V 9)$, thickness of the girder laminates $(V 2, V 3, V 10, V 11)$, length of the roving at the main girder $(V 4, V 5, V 6, V 7)$, and thickness of the roving laminate $(V 8)$. Fig. 8 presents parameterization of the model, whereas Table 1 contains box constraints imposed on each design variable.

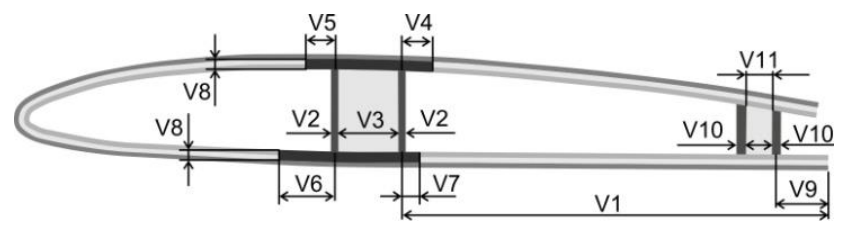

Fig. 8 Parameterization of the model of the UAV wing 
Limitations of the design variables

\begin{tabular}{|c|c|}
\hline Design variable & Range $[\mathrm{mm}]$ \\
\hline$V 1$ (position of the main girder) & $<118 \div 138>$ \\
\hline$V 2$ (thickness of the carbon fabric) & $<0.1 \div 0.48>$ \\
\hline$V 3$ (thickness of the foam PUR) & $<5 \div 25>$ \\
\hline$V 4-V 7$ (length of the roving) & $<2 \div 20>$ \\
\hline$V 8$ (thickness of the roving) & $<0.1 \div 2>$ \\
\hline$V 9$ (position of the small girder) & $<30 \div 40>$ \\
\hline$V 10$ (thickness of the glass fabric) & $<0.05 \div 1>$ \\
\hline$V 11$ (thickness of the foam PUR) & $<2 \div 10>$ \\
\hline
\end{tabular}

The goal of the optimization is to improve mechanical properties (reduce stresses, increase the stiffness) and reduce the total mass of the wing. The numerical model is fixed in all degrees of freedom on the one side and loaded on the top surface by distributed load equal to $5 \mathrm{kPa}$ (Fig. 9).

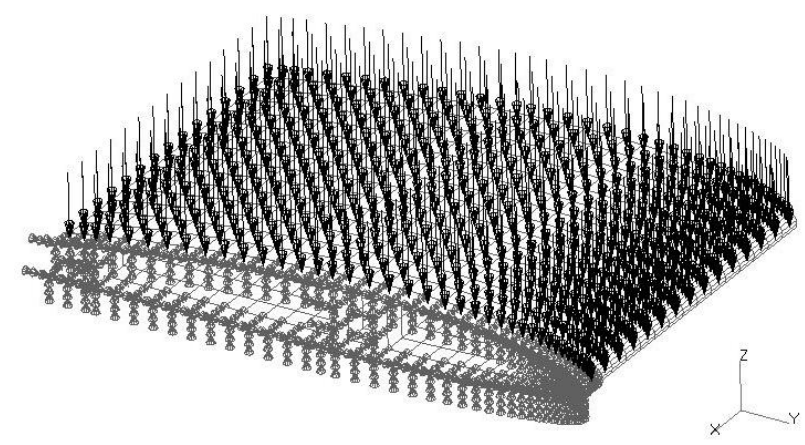

Fig. 9 Boundary conditions

In the initialization part and in the main loop of the algorithm for each individual, the fitness functions have to be calculated. Three following functionals for the optimization of the part of the wing are formulated:

- the minimization of the volume of the structure:

$$
\min _{x} \quad f_{1} \stackrel{d e f}{=} \int_{\Omega} d \Omega
$$

- the minimization of the maximal value of the equivalent (Von Mises) stress in the structure

$$
\min _{x} \quad \stackrel{\operatorname{def}}{=} f_{2} \max \left(\sigma_{e q}\right)
$$

- the minimization of the maximal value of vertical displacement (component uz) in the structure

$$
\min _{x} \quad \stackrel{\text { def }}{f_{3}}=\max \left(u_{z}\right) .
$$

Values of each functionals are calculated on the basis of results obtained from the numerical analyses. As mentioned before MSC Software CAE system are used to solve the problem. This system consists of modules: pre- and postprocessor Patran, and solver Nastran. In order to solve multi-objective optimization task by means of such CAE system, the creation of a geometrical and numerical model as well as the calculation of the fitness functional have to be fully automated. Thousands of fitness function calculations have to be performed in the particular multi-objective optimization task. Due to this, execution this step in a efficient way is crucial. It is done by means of additional ad-hoc software and appropriate scripts in PCL (Patran Command Language), which is implemented in module Patran [23]. For each separate fitness function calculation, the following steps have to performed. The 2D geometry of the model is generated on the basis eleven design variables. Than 3D surface model is generated, including definition of the materials and properties of each part of the model. Next boundary condition is defined and the finite element mesh is generated. After applying all necessary settings of the analysis input file for Nastran is generated. After solving the boundary value-problem, the fitness function is calculated by means of PCL and $\mathrm{C}++$ additional procedures. All communication steps between pre/post processor Patran, solver Nastran and MOOPTIM algorithm are realized via files. The steps of pre and post processing (Patran) are performed without invoking GUI (Graphical User Interface) of the system, but in the background mode. It allows to significantly reduce the time of the computation.

The idea of the entire multi-objective system is presented in Fig. 10. The algorithm MOOPTIM works until the stop condition is not fulfilled. It is defined as a maximum number of generation

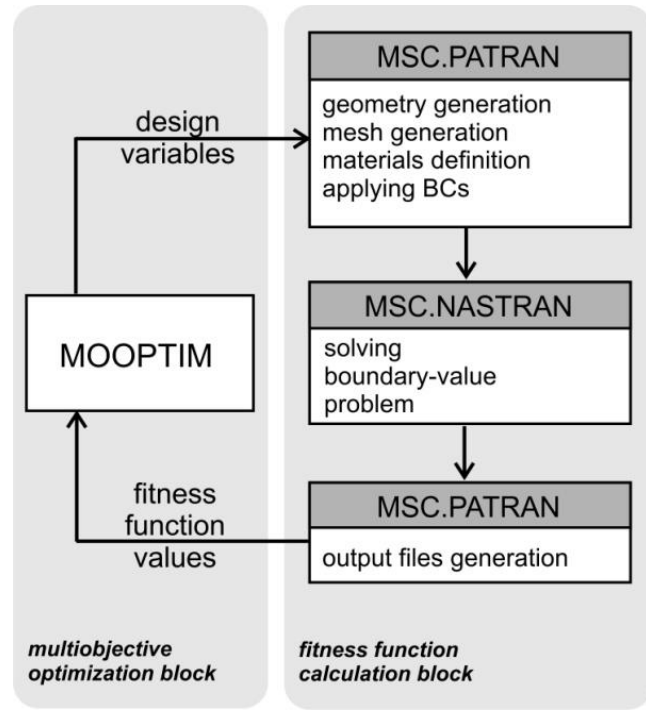

Fig. 10 Flowchart of the optimization system

\section{Results of the multi-objective optimization problem}

The multi-objective optimization concerns determining eleven design variables $(V 1-V 11)$, which minimize three defined functionals. Following variants (separate runs of optimization task) of the multi-objective optimization problems are performed:

- simultaneously minimization of the functionals (5) and (7);

- simultaneously minimization of the functionals (5) and (6);

- simultaneously minimization of the functionals (6) and (7);

- simultaneously minimization of all functionals (5), (6) and (7).

MOOPTIM algorithm has been run for the: probability of uniform mutation is equal to 0.1 , probability of Gaussian mutation is equal to 0.8 , probability of simple and arithmetic crossover is equal to 0.25 . The population size 
and number of generations are equal to 50 for the 2-objective optimization variants, whereas for the 3-objective optimization variant the population size is equal to 150 and number of generations equal to 100 .

Results of the bi-objective optimization for minimization of different pairs of functionals are presented in Figs. 11-13. Each graph presents obtained Pareto front with comparison to the solution for existing design. Existing design is understood as a structure of the UAV wing, described and modeled in Chapter 2. Obtained Pareto optimal solutions are mutually contradictory. Improving one of the functional another deteriorates. It can be easily observed, that obtained results strongly dominates existing design. For each optimization task, values of the functionals can be significantly improved.

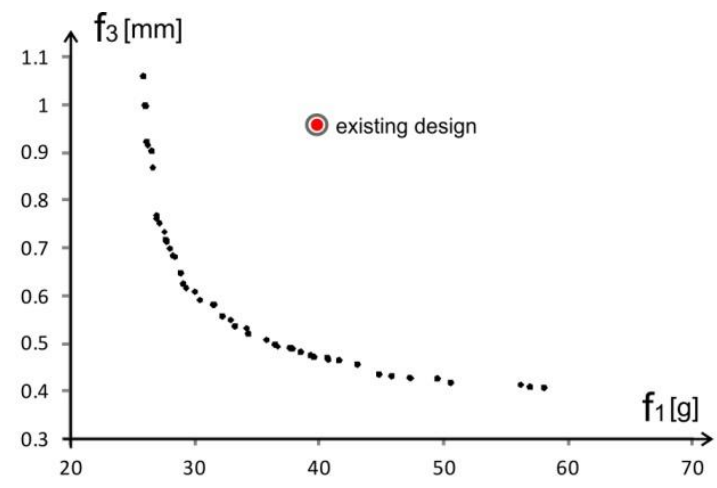

Fig. 11 Result of the bi-objective optimization for minimization of the functionals $f_{1}$ and $f_{3}$

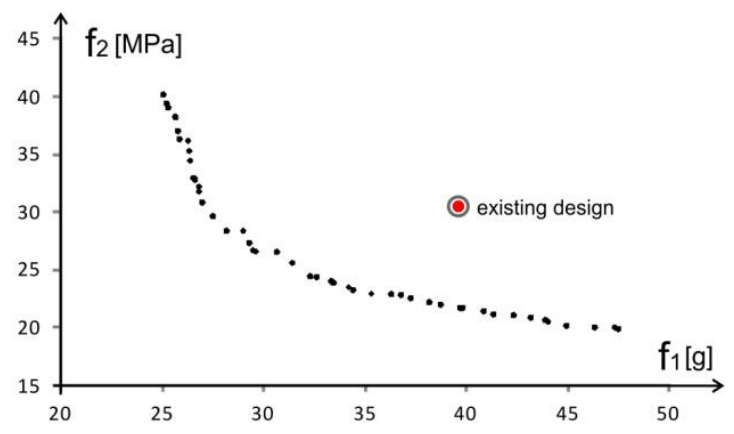

Fig. 12 Result of the bi-objective optimization for minimization of the functionals $f_{1}$ and $f_{2}$

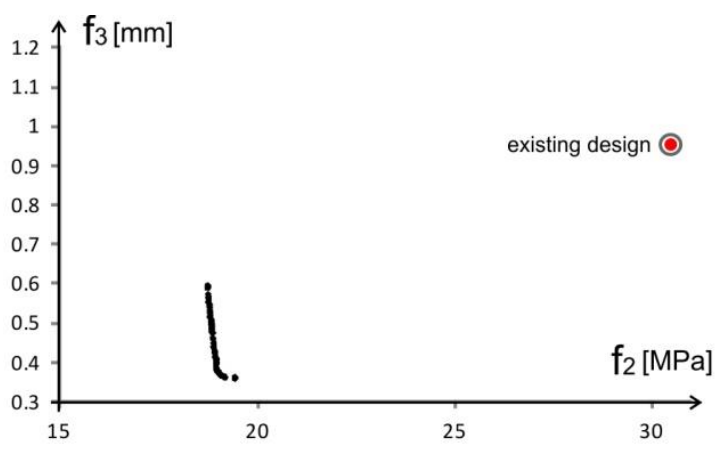

Fig. 13 Result of the bi-objective optimization for minimization of the functionals $f_{2}$ and $f_{3}$

Fig. 14 presents 3-dimensional Pareto front for the simultaneously optimization of all three functionals. Additionally five point are indicated in Fig. 14:

- solution for the existing design;

- point $\mathrm{A}$ - solution for the minimal value of the functional $f_{1}$;

- point B - solution for the minimal value of the functional $f_{2}$; functional $f_{3}$

- point $\mathrm{C}$ - solution for the minimal value of the

- point $\mathrm{D}$ - compromise solution located in the region close to the utopia (ideal) point -bottom left corner of the box which contains Pareto-optimal solutions.

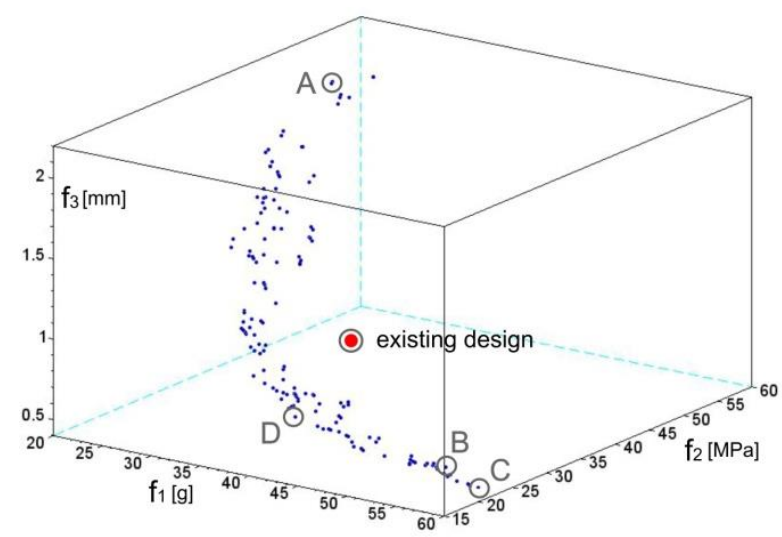

Fig. 14 Set of Pareto-optimal solutions for the simultaneously minimization of all functionals $\left(f_{1}, f_{2}\right.$ and $\left.f_{3}\right)$

Table 2

Values of the design variables and functionals for the existing and selected designs

\begin{tabular}{|c|c|c|c|c|c|}
\hline Design variable/ Functional & Existing design & Point A & Point B & Point C & Point D \\
\hline \hline$V 1$ (position of the main girder) & $128.88 \mathrm{~mm}$ & $131.77 \mathrm{~mm}$ & $133.82 \mathrm{~mm}$ & $126.56 \mathrm{~mm}$ & $134.87 \mathrm{~mm}$ \\
\hline$V 2$ (thickness of the carbon fabric) & $0.16 \mathrm{~mm}$ & $0.17 \mathrm{~mm}$ & $0.48 \mathrm{~mm}$ & $0.34 \mathrm{~mm}$ & $0.21 \mathrm{~mm}$ \\
\hline$V 3$ (thickness of the foam PUR) & $15 \mathrm{~mm}$ & $5 \mathrm{~mm}$ & $21.84 \mathrm{~mm}$ & $18.51 \mathrm{~mm}$ & $5.72 \mathrm{~mm}$ \\
\hline$V 4$ (length of the roving) & $10 \mathrm{~mm}$ & $15.88 \mathrm{~mm}$ & $19.73 \mathrm{~mm}$ & $17.47 \mathrm{~mm}$ & $16.71 \mathrm{~mm}$ \\
\hline$V 5$ (length of the roving) & $8 \mathrm{~mm}$ & $19.63 \mathrm{~mm}$ & $15.48 \mathrm{~mm}$ & $20 \mathrm{~mm}$ & $19.2 \mathrm{~mm}$ \\
\hline$V 6$ (length of the roving) & $15 \mathrm{~mm}$ & $12.14 \mathrm{~mm}$ & $18.52 \mathrm{~mm}$ & $20 \mathrm{~mm}$ & $10.7 \mathrm{~mm}$ \\
\hline$V 7$ (length of the roving) & $10 \mathrm{~mm}$ & $20 \mathrm{~mm}$ & $8.07 \mathrm{~mm}$ & $14.21 \mathrm{~mm}$ & $4.76 \mathrm{~mm}$ \\
\hline$V 8$ (thickness of the roving) & $1.2 \mathrm{~mm}$ & $0.1 \mathrm{~mm}$ & $1.83 \mathrm{~mm}$ & $1.99 \mathrm{~mm}$ & $1.19 \mathrm{~mm}$ \\
\hline$V 9$ (position of the small girder) & $34.99 \mathrm{~mm}$ & $36.093 \mathrm{~mm}$ & $36.59 \mathrm{~mm}$ & $38.57 \mathrm{~mm}$ & $36.59 \mathrm{~mm}$ \\
\hline$V 10$ (thickness of the glass fabric) & $0.1 \mathrm{~mm}$ & $0.05 \mathrm{~mm}$ & $0.31 \mathrm{~mm}$ & $0.93 \mathrm{~mm}$ & $0.41 \mathrm{~mm}$ \\
\hline$V 11$ (thickness of the foam PUR) & $4 \mathrm{~mm}$ & $2.42 \mathrm{~mm}$ & $9.36 \mathrm{~mm}$ & $9.9 \mathrm{~mm}$ & $8.1 \mathrm{~mm}$ \\
\hline \hline Mass functional $f_{1}$ - equation $(5)$ & $39.66 \mathrm{~g}$ & $23.37 \mathrm{~g}$ & $57.41 \mathrm{~g}$ & $57.84 \mathrm{~g}$ & $34.43 \mathrm{~g}$ \\
\hline Stress functional $f_{2}$ - equation $(6)$ & $30.49 \mathrm{MPa}$ & $57.03 \mathrm{MPa}$ & $18.98 \mathrm{MPa}$ & $23.13 \mathrm{MPa}$ & $26.64 \mathrm{MPa}$ \\
\hline Displacement functional $f_{3}$ - equation $(7)$ & $0.96 \mathrm{~mm}$ & $1.92 \mathrm{~mm}$ & $0.57 \mathrm{~mm}$ & $0.40 \mathrm{~mm}$ & $0.67 \mathrm{~mm}$ \\
\hline
\end{tabular}



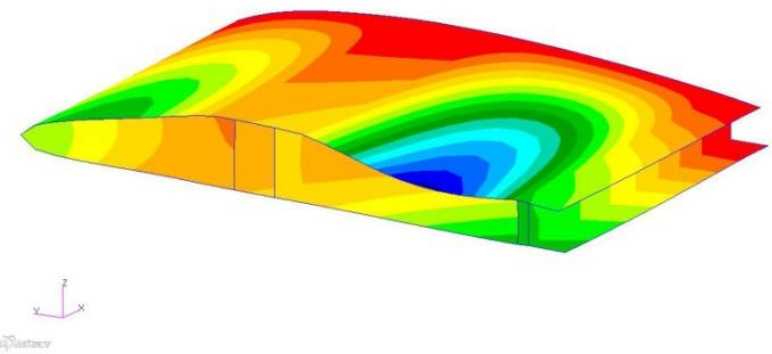
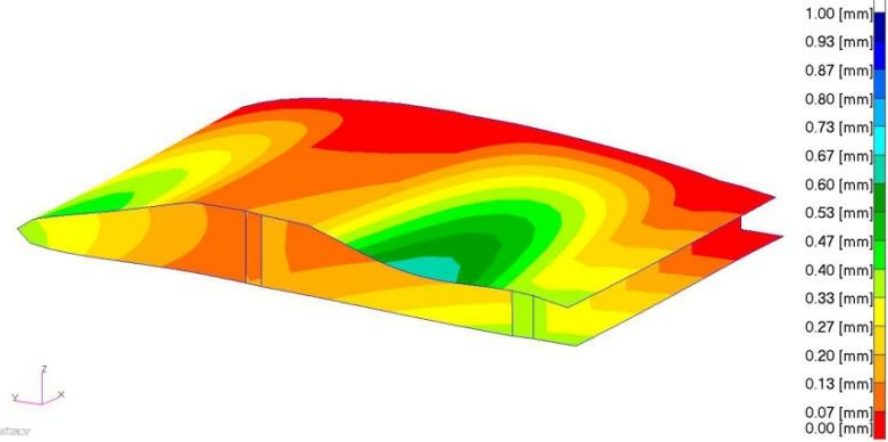

b

Fig. 15 Comparison of the vertical displacement between existing design and exemplary compromise solution (point D)

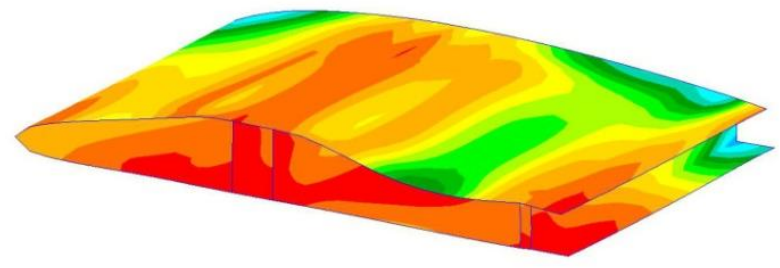

$\underbrace{2} \times$

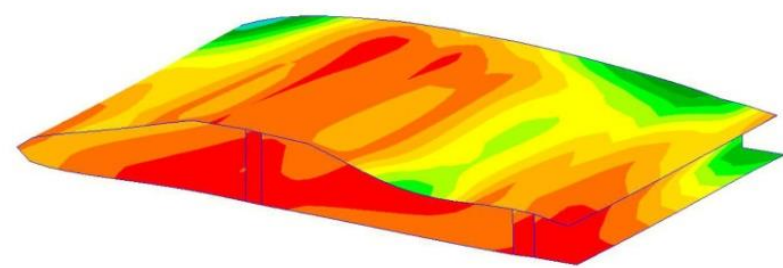

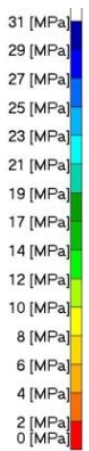

a

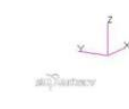

(2)

Fig. 16 Comparison of the Von Mises stresses between existing design and exemplary compromise solution (point D)

As for the bi-objective optimization tasks, for the 3-objective optimization, existing design is dominated by obtained Pareto optimal solutions. There are exists many solutions, for which comparing to the existing design, all functionals can be simultaneously improved. As an example, for point $\mathrm{D}$, mass can be reduced about $13 \%$, while maximal value of the equivalent stress and maximal displacement can be reduced about $12 \%$ and $30 \%$ respectively. For the 2 -objective optimization tasks, improvement of functionals with respect to existing design is much higher (Fig. 11, Fig. 12 and Fig. 13). Detailed comparison between value of the functionals and values of the design variables, for all point indicated in Fig. 14, are collected in Table 2. Fig. 15 and Fig. 16 presents map of vertical displacement and map of equivalent stress for the existing design and the compromise solution at point $\mathrm{D}$.

\section{Final remarks}

The designing of the UAV wing, taking into consideration more criteria, belongs to difficult optimization tasks. Intuitive solutions can be found only for a simple cases and usually it is far from optimal solution. In order to improve existing proposals and find the best solution with respect to design assumptions, efficient method of multi-objective optimization is needed. The multi-objective optimization of the UAV wing based on evolutionary computation has been presented. In-house implementation of the algorithm (MOOPTIM) has been utilized for the multi-objective optimization problem. Numerical model of the UAV wing, composed of different laminate materials has been prepared and verified experimentally. Three different criteria have been proposed and defined. Additional, different optimization functionals can be easily formulated and implemented.
Values of the functionals are solved by means of FEM. The preparation of the separate boundary-value problems is fully automated by means of in-house scripts and procedures. The model of the UAV wing has been parameterized using eleven design variables. Separate 2-objective and 3-objective optimization problems have been solved. The optimization results have been presented in form of Pareto frontiers of non-dominated solutions. The results of optimization have been compared with the existing solution for the wing (model of the real UAV wing). For each optimization task, obtained results strongly dominates existing design. It allows for reduction of the mass of the structure with simultaneously improving mechanical properties (increase stiffness and decrease stresses in the model). Application of the MOOPTIM algorithm and presentation of the results of the optimization as a set of 2D or 3D Pareto frontier, can significantly improve design process of the aircraft wing.

\section{References}

1. Collette, Y.; Siarry, P. 2003. Multiobjective Optimization: Principles and Case Studies, Springer, 293 p.

2. Coello, C.A. 2015. Recent trends in evolutionary multiobjective optimization, In: Evolutionary Multiobjective Optimization: Theoretical Advances And Applications, 7-32.

http://dx.doi.org/10.1007/1-84628-137-7_2.

3. Berci, M.; Toropov, V.; Hewson, R.; Gaskell, P. 2014. Multidisciplinary multifidelity optimisation of a flexible wing aerofoil with reference to a small UAV, Structural and Multidisciplinary Optimization 50: 683-699. http://dx.doi.org/10.1007/s00158-014-1066-2.

4. Ceruti, A.; Caligiana, G.; Persiani, F. 2013. Comparative evaluation of different optimization methodologies 
for the design of UAVs having shape obtained by hot wire cutting techniques, International Journal on Interactive Design and Manufacturing 7: 63-78.

http://dx.doi.org/10.1007/s12008-012-0164-x.

5. Lam, X.B.; Kim, Y.S.; Hoang, A.D.; Park, C.W. 2009. Coupled aerostructural design optimization using the kriging model and integrated multiobjective optimization algorithm, Journal of Optimization Theory and Applications 142: 533-556. http://dx.doi.org/10.1007/s10957-009-9520-9.

6. Beluch, W.; Burczynski, T.; Kus, W. 2010. Parallel artificial immune system in optimization and identification of composite structures, Proc In: Parallel Problem Solving from Nature, 11th International conference, Kraków, Poland, Berlin: Springer, 171-180. http://dx.doi.org/10.1007/978-3-642-15871-1_18.

7. Honda, S.; Igarashi, T; Narita, Y. 2013. Multi-objective optimization of curvilinear fiber shapes for laminated composite plates by using NSGA-II, Composites: Part B 45: 1071-1078. http://dx.doi.org/10.1016/j.compositesb.2012.07.056.

8. Ogierman, W.; Kokot, G. 2015. Modeling of constitutive behavior of anisotropic composite material using multi-scale approach, Mechanika 21(2): 118-122. http://dx.doi.org/10.5755/j01.mech.21.2.10276.

9. Omkar, S.N.; Senthilnath, J.; Khandelwal, R.; Narayana Naik, G.; Gopalakrishnan, S. 2011. Artificial Bee Colony $(\mathrm{ABC})$ for multi-objective design optimization of composite structures, Applied Soft Computing 11: 489-499. http://dx.doi.org/10.1016/j.asoc.2009.12.008.

10. Poteralski, A.; Szczepanik, M.; Beluch, W.; Burczynski, T. 2014. Optimization of composite structures using bio-inspired methods, Proc In: Artificial intelligence and soft computing. ICAISC 2014. 13th International conference, Zakopane, Poland, June 1-5, (eds. L. Rutkowski, et al.). Berlin, Springer: 385-395. http://dx.doi.org/10.1007/978-3-319-07176-3_34.

11. Chenchao Xia; Weifang Chen 2015. Gradient-based aerothermodynamic optimization of a hypersonic wing profile, Proc. In: 7th International Conference on Fluid Mechanics, ICFM7, Elsevier Procedia Engineering 126: 189-193.

http://dx.doi.org/10.1016/j.proeng.2015.11.214.

12. Ziqiang, Z.; Hongyan, F.; Rixin, Y.; Jie, L. 2004. Multi-objective optimization design of airfoil and wing, Science in China Ser. E Technological Sciences 47 (1): 15-25. http://dx.doi.org/10.1360/02ye0350.

13. Dlugosz, A.; Burczynski, T. 2012. Multiobjective shape optimization of selected coupled problems by means of evolutionary algorithms, Bulletin of the Polish Academy of Sciences, Technical Sciences. 60(2): 215222. http://dx.doi.org/10.2478/v10175-012-0028-3.

14. Zienkiewicz, O.C.; Taylor, R.L. 2000. The Finite Element Method, vol. 1-3, Butterworth, Oxford, 689 p.

15. Gay, D. 2014. Composite Materials: Design and Applications, Third Edition. CRC Press, 635 p.
16. Gibson, R.F. 2012. Principles of Composite Material Mechanics, CRC Press, 683 p.

17. Chung, D.L. 2010. Composite Materials: Science and Application, Springer-Verlag London. http://dx.doi.org/10.1007/978-1-84882-831-5.

18. Jones, R.M. 1998. Mechanics of Composite Materials. Materials Science and Engineering Series. Taylor \& Francis, 538 p.

19. Kleiber, M. 1998. Handbook of Computational Solid Mechanics, Springer-Verlag, Berlin, 763 p. http://dx.doi.org/10.1007/978-3-642-80396-3.

20. Ogierman, W.; Kokot, G. 2016. A study on fiber orientation influence on the mechanical response of a short fiber composite structure, Acta Mech 227: 173-183. http://dx.doi.org/10.1007/s00707-015-1417-0.

21. Deb, K. 1999. Multi-objective Genetic Algorithms: Problem Difficulties and Construction of Test Problems, Evolutionary. Computation. 7(3): 205-230. http://dx.doi.org/10.1162/evco.1999.7.3.205.

22. Deb, K.; Pratap, A.; Agarwal, S.; Meyarivan, T. 2002. A fast and elitist multi-objective genetic algorithm: NSGA-II, IEEE T. Evolut. Comput. 6(2): 181197. http://dx.doi.org/10.1109/4235.996017.

23. MSC.Patran/Nastran documentation 2013, MSC Software Corporation.

A. Długosz, W. Klimek

\section{MULTI-OBJECTIVE OPTIMIZATION OF THE UAV WING BY MEANS OF EVOLUTIONARY COMPUTATIONS}

S u m m a r y

The paper presents an application of the in-house implementation of the evolutionary multi-objective algorithm. Different types of functionals, which depend on equivalent stress, displacement and total mass of the structure are defined. Values of the functionals are calculated on the basis of results obtained from numerical simulations. Numerical model of the UAV wing, composed of different laminate materials has been prepared and verified experimentally. Automatic calculation of the fitness functionals for the parameterized model is prepared. Examples of multiobjective optimization by means of 2D and 3D Pareto-optimal set of solutions are presented. Effectiveness and usefulness of proposed method of multi-objective optimization are shown.

Keywords: multi-objective optimization, evolutionary algorithms, composite materials, finite element method, UAV wing.

Received March 20, 2016

Accepted November 25, 2016 